\title{
The Research on Compression Image Quality of Full Field Digital Mammography on PACS Environment
}

\author{
Jaeho Jeong*, Eunsoo Kim ${ }^{* *}$ \\ Department of Radiologic Science, Kyung Hee Medical Center ${ }^{*}{ }^{* *}$ \\ Department of Electronics, Kwangwoon University, ${ }^{*}{ }^{* *}$

\section{PACS환경에서 Full Field Digital Mammography 영상의 압축 화질평가에 관한 연구} \\ 정재호*, 김은수** \\ 경희의료원 $\mathrm{PACS}$ 팀, 광운대학교 ${ }^{*}$ **
}

\begin{abstract}
We tried to assessment about characteristics of image through quantitative evaluation method and qualitative evaluation method in Full Field Digital Mammography. It satisfied an approval standard of ten score regardless of compression ratio measuring detection score after compressing and appling an algorithm of JPEG2000 orJPEG compression targeting ACR accreditation phantom. Also, it was apparent that when we selected and compressed the image of real fine lesion and measured a change of diagnosis ability magnifing over 50 percent after compressing over 20:1 ratio, it had a strong influence on diagnosis ability. We realized that the difference between the original image according to compression ratio measuring a quantitative evaluation which is PSNR,RMSE,MAE and SSIM was relatively allowable.
\end{abstract}

Key Words : Full Field Digital Mammography, quantitative evaluation, qualitative evaluation, PSNR

\section{요 야}

디지털 Mammography영상에서 압축률에 따른 화질의 특성에 대해 정량적 평가와 정성적평가를 실시하였다. ACR Accreditation phantom을 대상으로 JPEG2000, JPEG 압축알고리즘을 적용하여 압축한 후 Detection Score를 측정하 여 압축률에 상관없이 10 점 인정기준을 충족하였다. 또한 실제미세병소 영상을 선정하여 압축을 실시하여 확대율에 따른 진단능의 변화를 측정하여 $20: 1$ 이상 압축 후 $50 \%$ 이상 확대 시 진단능에 영향을 미칠 수 있음을 알 수 있었다. 또한, 정량적 평가방법인 PSNR, RMSE, MAE, SSIM등을 측정하여 압축률에 따른 원본영상과의 차이점은 비교적 허 용 가능한 오차범위 내의 값이 측정되었다. 또한 MTF 측정을 통해 Full Field Digital Mammography 영상의 화질이 진단에 적합한 영상임을 알 수 있었다.

중심 단어: 디지털유방촬영장치, 정성적평가, 정량적평가, PSNR, MTF

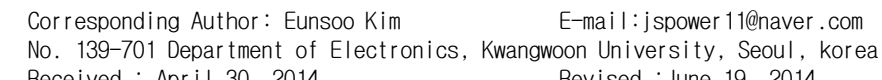

Tel: $+82-32-322-8219$ 


\section{I . INTRODUCTION}

유방엑스선영상은 촉진으로 진단하기 힘든 미세 석 회화 병변이나 초기의 유방암을 진단할 수 있는 진단 도구이다 ${ }^{[1]}$. 2009년 미국의 Cancer statistics에 따르면 유방암이 여성의 암 발병률 1 위, 사망률 2 위로 여성에 있어 가장 흔한 악성종양으로서 점차 증가하는 추세 이다 ${ }^{[2]}$. 유방암 조기 진단을 위해 자가 촉진 이외에 Screening 검사로 Mammography가 널리 사용되고 있다. Full-Field Digital Mammography (FFDM)는 전반적인 화 질, 피부와 유두, 대흥근의 묘사, 미세석회화 표현등 기존의 screen-film mammography(SFM) 타입보다 뛰어 나다 ${ }^{[3]-[6]}$. 본 논문에서 Picture Archiving Communi cations System (PACS)가 설치된 의료기관에서 FullField Digital Mammography (FFDM) 검사 후 PACS에 저 장할 때 Table 2에서와 같이 각 의료기관에서 객관적 기준 없이 임의의 방법으로 압축률과 압축알고리즘을 적용하고 있음을 조사를 통해 알 수 있었다. 대부분의 의료기관에서는 JPEG 압축을 선택하여 적용하고 있었 고 일부 소수의 의료기관에서 JPEG 2000 압축을 적용하 고 있었다. JPEG2000 알고리즘은 코사인 함수를 기반 으로 하는 DCT(Discrete Cosine Transform)대신 웨이블 릿 함수를 기반으로 하는 DWT(Discrete wavelet transform)를 사용하여 ${ }^{[7]}$ 블록화 현상이 발생하지 않으 므로 저속의 비트율에서도 뛰어난 품질의 영상을 제 공하여 뛰어난 압축 성능을 가지고 있다. 이에 압축률 및 압축방법에 따른 임상적 영향을 정성적, 정량적 측 정법을 통해 의료영상의 화질의 변화에 대한 데이터 정보를 구축하여 의료영상의 압축 영향 평가기준을 제시 하고자 하였다. 또한 평가된 방법으로 실제 초미 세병변 검출영상을 선택하여 확대율 $(0 \%, 50 \%, 100 \%)$ 에 따른 영상의 화질비교를 실시하였다. 또한 Full field digital mammography의 정량분석을 위해 International Electro technical Commission (IEC; 62220-1-2)의 규정에 따라 Edge method를 이용한 Module Transfer Function(MTF)를 측정하였다. 일반적으로 PACS환경 에서 디지털의료영상의 압축에 사용하는 JPEG $2 \mathrm{~K}$ 압 축과 JPEG 압축률의 변화에 따른 화질의 변화 및 노 이즈 발생 영향등을 분석하여 적정 압축률을 제시하 고자 한다.

\section{EXPERIMENTAL AND METHOD}

\section{ACR Accreditation phantom 실험}

본연구에서 사용한 검사장치는 2011년 도입된 Senographe DS(GE healthcare, Milwaukee, Wisconsin, USA)이며 실험 전 장치의 품질관리기준을 이수 하였 다. 지방조직과 유선조직 50:50의 비율로 구성된 두께 $4.2 \mathrm{~cm}$ ACR Accreditation image quality phantom(FLUKE Biomedical, 18-220)을 이용하여 촬영하였다.

\section{1 정량적 화질평가}

Peak Signal to Noise Ratio: PSNR, Root Mean Squared Error: RMSE, Mean Absolute Error: MAE, Structural SIMilarity: SSIM등을 측정하여 상대적 평가를 실시한 다.

Raw 데이터와 압축영상간의 왜곡측정을 위해 Image $\mathrm{J}$ 를 이용하여 평가하였으며 먼저 JPEG 2000 알고리즘을 이용한 압축영상의 화질이 Raw 데이터와 노이즈발생 정도를 평가하기 위해 Signal to Noise Ratio(SNR)를 구 하였다. PSNR(Peak Signal to Noise Ratio)은 임상적으로 시각적인 차이를 $\mathrm{db}$ 로 표현하기 위해 측정하였다 ${ }^{[8]}$

$$
P S N R=10 \cdot \log _{10}\left(\frac{M A X_{I}^{2}}{M S E}\right)=20 \cdot \log _{10}\left(\frac{M A X_{I}}{\sqrt{M S E}}\right)
$$

일반적으로 PSNR이 $30 \mathrm{~dB}$ 이상이면 임상적으로 영상 의 질적 차이를 구분할 수 없고, PSNR이 높을수록 영 상의 질이 좋게 평가 된다.

Raw 데이터와 압축영상간의 같은 양의 데이터에 대 해 동일한 위치에 대해 표준편차를 계산하기 위해 RMSE(Root Mean Square Error)를 측정하였다.

$$
\mathrm{SE}=\sqrt{\frac{1}{\mathrm{~N}} \sum_{\mathrm{i}=1}^{\mathrm{N}}\left(\mathrm{y}_{1}-\mathrm{X}_{1}\right)^{2}}
$$

$\mathrm{Raw}$ 데이터와 압축영상간의 오차들의 절대값의 평 균등을 측정하기 위해 $\mathrm{MAE}(\mathrm{Mean}$ Absolute Error)를 측 정하였다.

$$
M A E=\frac{1}{M N} \sum_{i=0}^{M} \sum_{j=0}^{N}|T(i, j)-I(i, j)|
$$

Full Reference 영상화질평가 방법 중 luminance 
change, contrast change, structural change를 감지할 수 있는 구조적 유사도(Structural SIMIlarity: SSIM)를 측정 하였다 ${ }^{[9]}$.

\section{2. 정성적 화질평가}

본 연구에서 사용된 Radiation Quality는 공칭 초점값 (nominal focal spot) 0.4 를 넘지 않는 조건에서 IEC 61267(Table 1.)에서 제정된 선질을 이용하였다. ACR Accreditation image quality phantom을 이용하여 촬영모 드는 AEC Standard를 선택하였으며 target 모드는 Molybdenum/Rhodium (Mo/Rh)선질을 선택하였다. 이 렇게 획득되어진 영상에 JPEG 2K, JPEG 압축알고 리즘 JPEG2000 영상 압축은 페가수스 키트(PICTools 32-Bit Imaging Development Kits, Pegasus Imaging Corporation, Tampa, FL. U.S.A)을 적용하여 표 1에서와 같은 비율로 압축을 실시한다. 판독 전문의 3 명이 각 각 압축된 영상의 Detection score를 작성하였다. 또한, 실제 미세질환 영상 5 장을 선택하여 관찰자 5 명이 각 영상에 대해 5회씩 관찰하여 그 평균값을 도출하였으 며 실험방법은 압축률 $(10: 1,20: 1,40: 1,60: 1,80: 1,100: 1)$ 과 확대율 $(50 \%, 100 \%)$ 의 차이에 대해 5 단계의 평가 (1 definitely unacceptable for diagnosis, 2: probably unacceptable, 3:equivocal, 4: probably acceptable, 5: definitely acceptable for diagnosis)를 실시하였다.

\section{Detector종류별 MTF측정을 통한 정량적 화질 평가}

방사선영상의 질(Quality)을 평가하기 위해 영상의 전 영역에 걸쳐 공간주파수(Spatial frequency : line pair $/ \mathrm{mm}$ )의 시스템 Performance에 대한 시스템 정보를 나타내는 정량분석의 척도가 되는 $\mathrm{MTF}$ 를 사용하였다 ${ }^{[10]} . \mathrm{MTF}$ 의 개념은 영상의 질 저하 정도를 정량적으로 나타내는 것이다. 변조(Modulation)라 함은 정보전달신 호의 진폭(amplitude) 또는 강도 (Intensity), 양(Amount) 의 변화를 의미한다. 또한 MTF는 픽셀 값들을 공간주 파수(Spatial Prequency)에 대해 영상 시스템의 전달능을 나타낸다. MTF는 주로 영상의 화질을 결정하는 인자 중 하나인 선예도를 평가하는 방법이다. 먼저 실험한 Edge영상을 가지고 Matlab을 이용하여 Edge Spread Function(ESF)을 구한다. ESF을 다시 미분하여 Line
Spread Function (LSF)을 구하고 다시 LSF를 Fast Fourler Transform (FFT)하여 영상시스템의 주파수 전 달 함수(Response function)인 MTF를 다음의 계산과정 을 거쳐 구하였다. 압축률에 따른 정량적 화질평가를 위해 디지털 mammography 영상장치를 대상으로 Table1.과 같이 Raw데이터를 각각 압축하였다.

Table 1. The compression ratio of compression on raw data

\begin{tabular}{cc}
\multicolumn{3}{c}{ Compression Type } \\
JPEG 2K & JPEG \\
\hline $10: 1$ & $7: 1$ \\
$20: 1$ & $8: 1$ \\
$40: 1$ & $11: 1$ \\
$60: 1$ & $20: 1$ \\
$80: 1$ & $37: 1$ \\
$100: 1$ & \\
\hline
\end{tabular}

Table 2. The compression ratio of Modilities in Hospital

\begin{tabular}{cccccc}
\hline 구분 & KH & AJ & EH & HK & KK \\
\hline \multirow{2}{*}{ CR } & JPEG 2K & JPEG LY & JPEG LS & JPEG LY & JPEG 2K \\
& $30: 1$ & $18: 1$ & $13: 1$ & $7: 1$ & $35: 1$ \\
\multirow{2}{*}{ MG } & JPEG 2K & JPEG LS & JPEG LY & JPEG LY & JPEG 2K \\
& $30: 1$ & $3.7: 1$ & $20: 1$ & $20: 1$ & $30: 1$ \\
\multirow{2}{*}{ CT } & JPEG LS & JPEG LS & JPEG LS & JPEG LS & JPEG LS \\
& $2.5: 1$ & $2.5: 1$ & $2: 1$ & $3: 1$ & $2.5: 1$ \\
\multirow{2}{*}{ MR } & JPEG LS & JPEG LS & JPEG LS & JPEG LS & JPEG LS \\
& $2.6: 1$ & $2.6: 1$ & $2: 1$ & $3.5: 1$ & $3.5: 1$ \\
\hline \multicolumn{4}{c}{$*$ * LY $=$ LOSSY, LOSSLESS $=$ LS }
\end{tabular}

그리고 유효 해상력 증가를 위해 실시하는 Magnification Mammography는 병변의 형상화와 감지력 을 향상시킨다 ${ }^{[11],[12]}$. Magnification Mammography는 미 세한 잠재적 병소의 유무를 확인 하는데 있어 이론적 으로 확립되어 있는 표준방법 ${ }^{[13]}$ 으로 일반 digital mammography와 마찬가지로 magnification한 후 각각 2 회 촬영하여 정량적 평가 방법인 $\mathrm{MTF}$ 를 측정하였다. 이때 $\mathrm{MTF}$ 측정을 위하여 각 장치의 제조회사에서 제 공하는 측정 프로그램은 Direct detector와 Indirect detector의 객관적 평가를 하는데 있어서 적절치 않고 기준 값이 달라 사용하지 않고 Edge method를 활용하 
여 IEC 62220-1-2에 따라 실험하였다 ${ }^{[14]}$

\section{RESULT}

본 연구에서 영상의 종류별 압축률의 영향을 알아 보기 위해 Mammo ACR Phanatom, 일반 Digital mammography, magnification mammography 영상을 대상 으로 각각의 압축률별 PSNR, RAMSE, MAE, SSIM등을 측정하였다. 그 결과 JPEG 2K 20:1 압축에서 PSNR값 이 ACR Phantom 36.9db로 mammo 55.8db, mammo magni 55.5db,보다 낮았으며 RMSE값은 ACR Phantom 이 55.2로서 5.02, 6.9를 나타낸 mammo, mammo magni 보다 현저히 높게 나타났다.ACR Phantom 영상이 JPEG $2 \mathrm{~K}$ 압축에서 mammography영상보다 수학적 왜곡도가 상대적으로 심한 것을 알 수 있었다. 하지만 그에 반 해 JPEG 압축 알고리즘은 Phantom 영상, mammography, magnification mammography 영상 모두에서 균일한 값을 나타내었다. SSIM 측정값은 압축률에 따른 유의미한 변화값은 도출되지 않았다.

Table 3. PSNR according to Compression ratio

\begin{tabular}{cccc}
\hline & \multicolumn{3}{c}{ PSNR } \\
\cline { 2 - 4 } 구분 & Phantom & mammo & $\begin{array}{c}\text { mammo } \\
\text { magni }\end{array}$ \\
\hline $10: 1$ & 38.2 & 57.863 & 56.384 \\
$20: 1$ & 36.9 & 55.795 & 55.458 \\
$40: 1$ & 36.855 & 52.21 & 51.719 \\
$60: 1$ & 36.826 & 50.869 & 49.92 \\
$80: 1$ & 36.801 & 50.014 & 49.103 \\
$100: 1$ & 36.783 & 49.505 & 48.633 \\
\hline
\end{tabular}

Table 4. RMSE according to Compression ratio

\begin{tabular}{cccc}
\hline & \multicolumn{3}{c}{ RMSE } \\
\cline { 2 - 4 } 구분 & Phantom & mammo & $\begin{array}{c}\text { mammo } \\
\text { magni }\end{array}$ \\
\hline $10: 1$ & 54.1 & 4.356 & 6.209 \\
$20: 1$ & 55.2 & 5.052 & 6.908 \\
$40: 1$ & 55.483 & 7.633 & 10.624 \\
$60: 1$ & 55.67 & 8.906 & 13.069 \\
$80: 1$ & 55.834 & 9.828 & 14.358 \\
$100: 1$ & 55.944 & 10.421 & 15.156 \\
\hline
\end{tabular}

Table 5. MAE according to Compression ratio

\begin{tabular}{cccc}
\hline & \multicolumn{3}{c}{ MAE } \\
\cline { 2 - 4 } 구분 & Phantom & mammo & $\begin{array}{c}\text { mammo } \\
\text { magni }\end{array}$ \\
\hline $10: 1$ & 26.4 & 2.802 & 2.602 \\
$20: 1$ & 27.789 & 3.771 & 3.065 \\
$40: 1$ & 28.264 & 5.113 & 5.212 \\
$60: 1$ & 28.547 & 5.762 & 6.41 \\
$80: 1$ & 28.745 & 6.189 & 7.026 \\
$100: 1$ & 28.891 & 6.466 & 7.445 \\
\hline
\end{tabular}

압축률별 모의병소 실험에서는 미세석회화(speck), 섬유소(fiber), 종괴(mass) 검출능력은 압축률에 따른 미 세한 변경은 있었으나 최대 JPEG 2K 100:1 압축, JPEG 41:1 압축에서도 허용기준 10점이상을 유지하였 다.

PSNR(JPEG 2K)

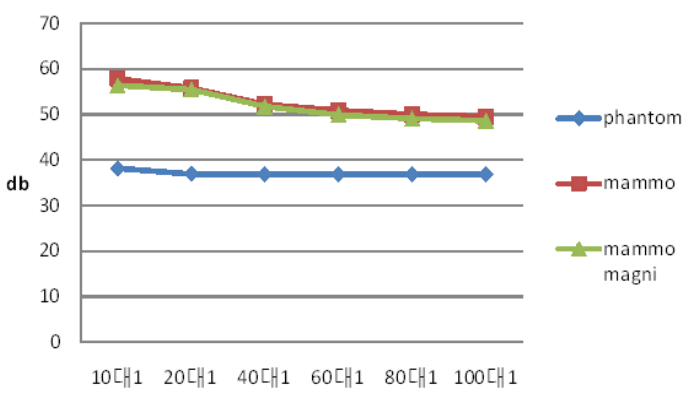

Fig. 1. PSNR according to Compression ratio(JPEG 2K).

PSNR(JPEG)

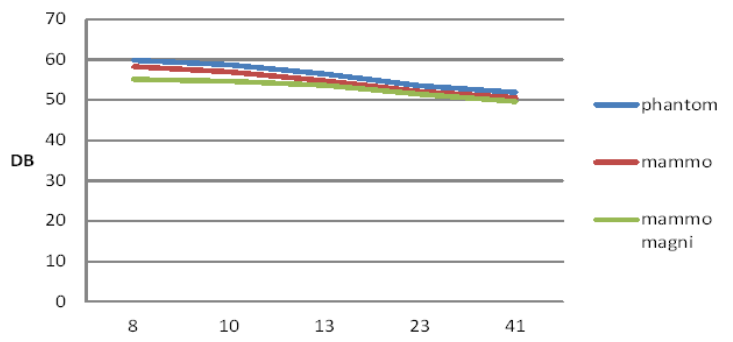

Fig. 2. PSNR according to Compression ratio(JPEG). 
"J. Korean Soc. Radiol., Vol. 8, No. 4, June 2014"

Table 6. Detection Scores for Test Phantom image according to Compression ratio(JPEG 2K)

\begin{tabular}{|c|c|c|c|c|c|c|c|}
\hline \multicolumn{2}{|c|}{ 구분 } & $10: 1$ & $20: 1$ & $40: 1$ & $60: 1$ & $80: 1$ & 100:1 \\
\hline \multirow{3}{*}{$\begin{array}{l}\text { Obser } \\
\text { ver } 1\end{array}$} & $\mathrm{Fib}$ & 5 & 5 & 5 & 4.5 & 4.5 & 4.5 \\
\hline & Spe & 5 & 3.5 & 3.5 & 3 & 3 & 3 \\
\hline & $\mathrm{Ma}$ & 3.5 & 4 & 4 & 4 & 3.5 & 3.5 \\
\hline \multicolumn{2}{|c|}{ Sum } & 13.5 & 12.5 & 12.5 & 11.5 & 11 & 11 \\
\hline \multirow{3}{*}{$\begin{array}{l}\text { Obser } \\
\text { ver } 2\end{array}$} & Fib & 5 & 4 & 4 & 4 & 4 & 4 \\
\hline & Spe & 4 & 3.5 & 4 & 3.5 & 3.5 & 3.5 \\
\hline & $\mathrm{Ma}$ & 4 & 4 & 4 & 3.5 & 3.5 & 3.5 \\
\hline \multicolumn{2}{|c|}{ Sum } & 13 & 11.5 & 12 & 11 & 11 & 11 \\
\hline \multirow{3}{*}{$\begin{array}{l}\text { Obser } \\
\text { ver } 3\end{array}$} & Fib & 4 & 5 & 4.5 & 4.5 & 4.5 & 4 \\
\hline & Spe & 5 & 3.5 & 3.5 & 3.5 & 3.5 & 3.5 \\
\hline & $\mathrm{Ma}$ & 3.5 & 4 & 4 & 4 & 3.5 & 3.5 \\
\hline \multicolumn{2}{|c|}{ Sum } & 12.5 & 12.5 & 12 & 12 & 11.5 & 11 \\
\hline
\end{tabular}

Table 7. Detection Scores for Test Phantom image according to Compression ratio(JPEG)

\begin{tabular}{|c|c|c|c|c|c|c|}
\hline \multicolumn{2}{|c|}{ 구분 } & $8: 1$ & $10: 1$ & $15: 1$ & 23:1 & $41: 1$ \\
\hline \multirow{3}{*}{$\begin{array}{l}\text { Obser } \\
\text { ver } 1\end{array}$} & Fib & 5 & 5 & 5 & 4.5 & 4.5 \\
\hline & Spe & 5 & 4 & 4 & 3.5 & 3.5 \\
\hline & $\mathrm{Ma}$ & 4 & 5 & 4 & 3.5 & 3.5 \\
\hline \multicolumn{2}{|c|}{ Sum } & 14 & 14 & 13 & 11.5 & 11.5 \\
\hline \multirow{3}{*}{$\begin{array}{l}\text { Obser } \\
\text { ver } 2\end{array}$} & Fib & 5 & 4 & 4 & 4 & 4 \\
\hline & Spe & 4 & 3.5 & 4 & 3.5 & 3.5 \\
\hline & $\mathrm{Ma}$ & 4 & 4 & 4 & 3.5 & 3.5 \\
\hline \multicolumn{2}{|c|}{ Sum } & 13 & 11.5 & 12 & 11 & 11 \\
\hline \multirow{3}{*}{$\begin{array}{l}\text { Obser } \\
\text { ver } 3\end{array}$} & $\mathrm{Fib}$ & 4 & 4.5 & 4 & 4.5 & 4 \\
\hline & Spe & 4.5 & 3.5 & 3.5 & 3 & 3.5 \\
\hline & $\mathrm{Ma}$ & 3.5 & 4 & 4 & 4 & 4 \\
\hline \multicolumn{2}{|c|}{ Sum } & 12 & 12 & 11.5 & 11.5 & 11.5 \\
\hline
\end{tabular}

실제미세병변영상 검출능력 실험에서는 확대하지 않은 원본영상일 때 진단능 평가 에서 100:1까지도 평 균 4.1 (probably acceptable)이상을 유지하였다. $50 \%$ 확대 영상에서는 20:1 압축에서 4.1(probably acceptable) 유지 하였으나 40:1이상 압축에서는 측정값 평균 3.2(equivocal) 판독 모호함으로 나타났다.
Table 8. The Test Score on Real micro disease image

\begin{tabular}{|c|c|c|c|c|c|c|}
\hline 구분 & $10: 1$ & $20: 1$ & $40: 1$ & $60: 1$ & $80: 1$ & $100: 1$ \\
\hline \multirow{5}{*}{$\begin{array}{l}0 \% \\
\text { magni }\end{array}$} & 4.8 & 4.4 & 4.4 & 4 & 4 & 4 \\
\hline & 5 & 4.4 & 4.2 & 4 & 4 & 4.2 \\
\hline & 4.8 & 4.2 & 4.2 & 3.8 & 4 & 4.2 \\
\hline & 4.4 & 4.2 & 4.2 & 3.8 & 3.6 & 3.8 \\
\hline & 5 & 4.2 & 4.2 & 4.2 & 3.6 & 4.2 \\
\hline \multirow{5}{*}{$\begin{array}{l}50 \% \\
\text { magni }\end{array}$} & 4.4 & 4.2 & 3.2 & 2.4 & 2.6 & 2.4 \\
\hline & 4.8 & 4 & 3 & 2.6 & 2.6 & 2.6 \\
\hline & 4.4 & 3.8 & 3.4 & 2.6 & 2.2 & 2.6 \\
\hline & 4.6 & 4.2 & 3.2 & 2.8 & 2.6 & 1.6 \\
\hline & 4.4 & 4.2 & 3 & 2.8 & 2.4 & 2.2 \\
\hline \multirow{5}{*}{$\begin{array}{c}100 \% \\
\text { magni }\end{array}$} & 4.6 & 3.8 & 2.6 & 2 & 1.8 & 1.6 \\
\hline & 4.4 & 4 & 3 & 2.2 & 2 & 1.8 \\
\hline & 4.4 & 3.8 & 3 & 2 & 1.6 & 1.8 \\
\hline & 4.4 & 3.8 & 3 & 1.8 & 1.6 & 1.4 \\
\hline & 4.2 & 3.8 & 2.8 & 2.2 & 2 & 1.8 \\
\hline
\end{tabular}

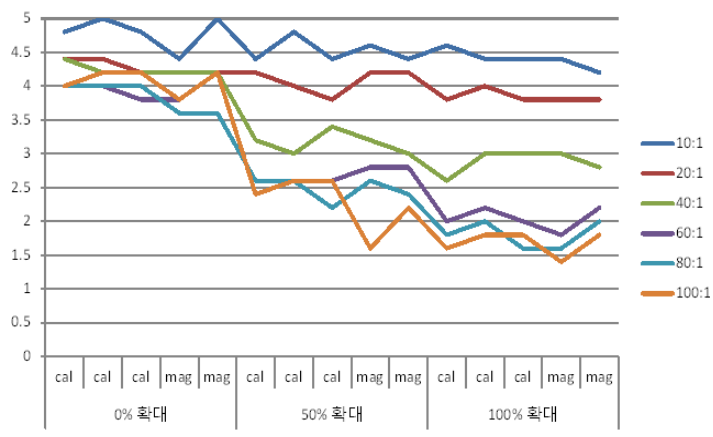

Fig. 3. The Test Score on Real micro disease image.

Direct Detector방식의 Senographe DS(GE)장비의 $\mathrm{MTF}$ 측정을 통한 정량적 화질평가는 Direct detector의 Mo target/Mo filtration에서의 MTF측정결과 MTF $0.1(10 \%)$ 의 값은 $5.5 \mathrm{Lp} / \mathrm{mm}$ 로 나타났으며 또한, Magnification mammography에서의 Mo/Mo, Direct detector MTF $0.1(10 \%)$ 의 값은 $5.2 \mathrm{Lp} / \mathrm{mm}$ 이다. 그리고 Magnification mammography에서의 Direct detector $\mathrm{Mo} / \mathrm{Rh}$ 에서의 MTF $0.1(10 \%)$ 값은 $5.3 \mathrm{Lp} / \mathrm{mm}$ 이다. 


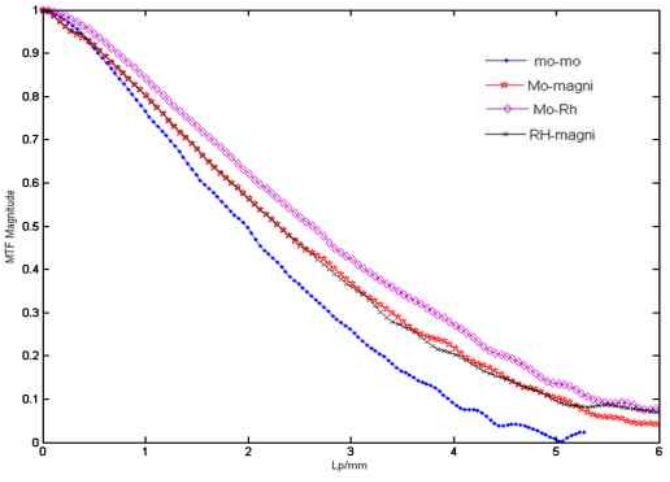

Fig. 4. MTF Measurement of Mo target/Mo filtration of FFDM.

\section{DISCUSSION}

Mammography 영상의 정량적 화질평가에서 JPEG $2 \mathrm{~K}$ 압축에서는 Fig. 1 에서처럼 압축률에 의한 변화보다 는 영상의 특성이 고주파영역이 많이 포함되며 미세 병소 부분이 많이 포함될수록 PSNR, RMSE, MAE 값 에서 영상의 수치적 왜곡이 많이 발생된다는 것을 알 수 있었다. 실제 미세병변영상의 평가에서는 JPEG $2 \mathrm{~K}$ $20: 1$ 영역에서는 $100 \%$ 확대율까지 진단 가능한 허용범 위이나 $40: 1$ 압축 이후부터는 $50 \%$ 영역부터 판독 모호 하거나 명확히 판독 불가한 영역으로 나타났다. 따라 서 압축률에 따른 진단능의 변화에서는 확대율 $1: 1$ 영 상일때는 판독에 별다른 지장이 없다고 추정되었으나 $50 \%$ 이상 확대 시 판독이 모호하거나 판독불가능 영 상의 양상을 보여 이에 대한 고려가 있어야 할 것으로 사료된다. Magnification mammography에서는 $\mathrm{Mo} / \mathrm{Mo}$ $5.2 \mathrm{Lp} / \mathrm{mm}, \mathrm{Mo} / \mathrm{Rh} 5.3 \mathrm{Lp} / \mathrm{mm}$ target 물질과 filtration 물질의 종류와 관계없이 비슷한 MTF값을 나타내어 확대촬영에서는 영상의 화질에 대한 변인요소가 줄어 드는 것을 알 수 있었다.일반 mammography 영상과 magnification mammography영상에서는 JPEG2k 압축에 서 PSNR수치는 최대 압축인 100대1에서도 비교적 우 수한 $49.5 \mathrm{~dB}, 48.6 \mathrm{~dB}$ 를 나타내어 압축에 따른 영상의 열화는 크지 않은 것으로 나타났으며 JPEG압축에서 $40: 1$ 압축에서도 $51.9 \mathrm{~dB}$ 로 측정되어 압축알고리즘에 의한 변인은 크지 않음을 알 수 있었다.

\section{CONCLUSION}

이상과 같은 내용으로 ACR Phantom을 이용한 모의 병소 실험을 통해 확대율에 따른 모의병소 검출능력 은 거의 변화가 없음을 인지하였고 압축률에 따른 정 량적 평가 수치 또한 허용범위 내였으나 다만, 고주파 영역의 영상 또는 미세한 병변영상 진단 시 노이즈발 생 또는 왜곡의 발생 가능성이 있음을 알 수 있었다.

Digital mammography의 실제 미세병변영상에 대한 진단 시에 영상의 확대율에 따라 압축률이 미치는 영 향이 매우 큼을 알 수 있었으며, 의료기관에서 압축률 지정 시 각 전문판독의의 확대 판독정도에 대한 정보 를 숙지하고 이에 대한 신중한 판독률을 설정하여야 하겠다.

\section{Reference}

[1] Sickles E.A., "Mammographic features of 300 consecutive nonpalpable breast cancers", AJR Am J Roentgenol, Vol. 146, pp. 661-663, 1986.

[2] Ahmedin Jemal, Rebecca Siegel, Elizabeth Ward, Yongping Hao, Jiaquan Xu, Michael J. Thun, "Cancer Statistics, 2009", CA Cancer J Clin, Vol. 59, pp. 225-249, 2009

[3] Fischmann A., Siegmann K.C., Wersebe A., Claussen C.D., Müller-Schimpfle M., "Comparison of full-field digital mammography and film-screen mammography: Image quality and lesion detection". Br J Radiol, Vol. 78, pp. 312-315, 2005.

[4] Karssemeijer N., Frieling J.T., Hendriks J.H., "Spatial resolution in digital mammography". Invest Radiol, Vol. 5, pp. 413-419, 1993.

[5] Harvey J.A., Bovbjerg V.E., "Quantitative assessment of mammographic breast density: relationship with breast cancer risk". Radiology, Vol. 230, pp. 25-27, 2004.

[6] Elmore J.G., Wells C.K., Lee C.H., Howard D.H., "Feinstein AR. Variability in radiologists'interpretations of mammograms", N Engl J Med, Vol. 331, pp. 1493-1499, 1994.

[7] Soonmu K, "Change of Image Quality within Compression of AAPM CT Performance Phantom Image Using JPEG2000 in PACS," Journal of The Korean Society of Radiology, vol. 6, no. 3, pp. 217-226, 2012.

[8] U-K Cho, J-H Hong, and S-B Cho"Image Enhancement based on the Genetic Algorithm for Reducing Impulsive Noises," 
Journal of Computing Science and Engineering, vol. 33, no. 1, pp. 283-285, 2006.

[9] Z. Wang et al., "Image Quality Assessment: from Error Visibility to Structural Similarity", IEEE Trans. Image Process., Vol. 13, No.4, pp. 600-612, Apr. 2004.

[10] Bosun Kang, "Development of Image Quality Evaluation Program for Diagnostic Radiography," Journal of The Korean Society of Radiology, vol. 2, no. 2, pp. 5-10, 2008.

[11] Bushberg J.T., Seibert J.A., Leidholdt E., et al. "The essential physics of medical imaging. 2nd ed. Philadelphia", PA: Lippincott Williams \& Wilkins, 2002.

[12] Hermann K.P., Obenauer S., Funke M., Grabbe E.H., "Magnification mammography: a comparison of full-field digital mammography and screen-film mammography for the detection of simulated small masses and microcalcifications". Eur Radiol., Vol. 12, pp. 2188-2191, 2002.

[13] Liu F., Kanal K.M., Stewart B.K., Lehman C.D., "Effects of Lesion Positioning on Digital Magnification Mammography Performance", Acad. Radiol. Vol. 17, pp. 791-794, 2010.

[14] Liu F., Kanal K.M., Stewart B.K., Lehman C.D., "Effects of Lesion Positioning on Digital Magnification Mammography Performance", Acad. Radiol. Vol. 17, pp. 791-794, 2010. 